\title{
Gestione della terapia adiuvante con mitotane nei pazienti affetti da carcinoma corticosurrenalico
}

\author{
Giuseppina De Filpo ${ }^{1}$. Letizia Canu ${ }^{1}$
}

Accettato: 13 febbraio 2020 / Pubblicato online: 25 agosto 2020

(c) The Author(s) 2020

\section{Introduzione}

Il carcinoma corticosurrenalico (adrenal cortical carcino$m a, \mathrm{ACC})$ è una patologia aggressiva e rara $(0,7-2$ casi per milione/anno) con picco di incidenza tra la quarta e la sesta decade [1]. Ad oggi l'intervento chirurgico radicale condotto da un chirurgo esperto rappresenta l'unico approccio curativo [2]. Il trattamento con mitotane trova indicazione nelle forme avanzate ed è ampiamente utilizzato anche in adiuvante, in quanto si è mostrato in grado di aumentare l'intervallo libero da malattia dopo la resezione chirurgica del tumore [3]. Un accurato follow-up dei pazienti sottoposti a terapia con mitotane è necessario al fine di monitorare i livelli plasmatici del farmaco e consentire un'adeguata gestione degli effetti collaterali.

\section{Indicazioni al trattamento}

Le recenti linee guida del 2018 raccomandano l'inizio della terapia adiuvante con mitotane (o,p'DDD), un derivato del pesticida dichloro-diphenyl-trichloroethane (DDT) introdotto a partire dagli anni '60 [4], nei pazienti ad alto rischio di ricorrenza di malattia (stadio III o R1, Ki67 >10\%), mentre non si esprimono a favore o contro per quanto riguarda il trattamento dei pazienti a rischio basso/intermedio (stadio I-II, R0, Ki67 $\leq 10 \%$ ) in cui la scelta viene valutata caso per caso [2]. Attualmente è in corso uno studio prospettico, randomizzato di fase III (ADIUVO), promosso dallo

$凶$ L. Canu

letizia.canu@unifi.it

1 Dipartimento di Scienze Biomediche Sperimentali e Cliniche "Mario Serio", Università di Firenze, Firenze, Italia
European Network of Adrenal Tumors (ENS@T), che prevede l'arruolamento dei pazienti con diagnosi di ACC a basso/intermedio rischio di ricorrenza di malattia randomizzati in due bracci: uno sottoposto a terapia adiuvante con mitotane e l'altro sottoposto al solo follow-up osservazionale. I dati derivanti dallo studio ADIUVO saranno in grado di fornire ulteriori indicazioni circa l'efficacia della terapia adiuvante con mitotane in questi pazienti in termini di sopravvivenza libera da malattia e globale.

\section{Monitoraggio e gestione degli effetti collaterali}

Il mitotane viene somministrato per os (Lysodren, compresse da 500 mg, HRA Pharma, Paris, France) ed è escreto per il $60 \%$ immodificato con le feci; il restante $40 \%$ si concentra nel fegato, nel SNC, nel tessuto adiposo e nelle ghiandole surrenaliche all'interno delle quali inibisce la steroidogenesi [5].

Per tale motivo, in tutti i pazienti trattati si rende indispensabile una terapia steroidea sostitutiva che può essere intrapresa contestualmente all'inizio della terapia con mitotane o entro le prime 2-3 settimane. Generalmente vengono somministrate dosi di glucocorticoidi maggiori rispetto a quelle utilizzate nei pazienti con insufficienza cortico-surrenalica a causa dell'aumento della cortisol binding protein (CBG) e dell' effetto di induzione sul CYP450 3A4, deputato al metabolismo dei glucocorticoidi, dovuti all'azione del mitotane [2, 6].

È possibile iniziare la somministrazione del mitotane con un regime a basse o alte dosi; una volta intrapresa la terapia, deve essere proseguita per almeno 2 anni e, se ben tollerata, per un massimo di 5 anni. In questo lasso di tempo il monitoraggio dei livelli plasmatici del farmaco è strettamente rac- 
Tabella 1 Parametri da monitorare in corso di terapia con mitotane [2]

\begin{tabular}{ll}
\hline Parametri & Frequenza \\
\hline Livelli plasmatici di mitotane & Ogni 3-4 settimane fino al raggiungimento di livelli $>14$ mg/L, successivamente ogni 2-3 mesi \\
AST, ALT, GGT, bilirubina & Inizialmente ogni 3-4 settimane per i primi 6 mesi, successivamente ogni 2-3 mesi \\
Emocromo & Inizialmente dopo 3-4 settimane, successivamente ogni 3-4 mesi \\
ACTH & Dosare nel sospetto di deficit o eccesso di glucocorticoidi \\
TSH, FT4 & Ogni 3-4 mesi \\
Renina & Ogni 6 mesi \\
Colesterolo totale, HDL, trigliceridi & Ogni 3-4 mesi, in terapia adiuvante \\
Testosterone totale, SHBG negli uomini & Ogni 3-4 mesi, in terapia adiuvante \\
\hline
\end{tabular}

comandato per valutare il raggiungimento e il mantenimento dei livelli della cosiddetta "finestra terapeutica" (14-20 $\mathrm{mg} / \mathrm{L})$ e per limitarne gli effetti avversi.

Il mitotane esercita un effetto limitato sulla glomerulare surrenalica; in genere, la necessità di instaurare una terapia mineralcorticoide dev'essere valutata sulla base del quadro clinico e biochimico [7].

Le linee guida raccomandano di monitorare la mitotanemia ogni 3-4 settimane fino al raggiungimento di livelli $>14$ $\mathrm{mg} / \mathrm{L}$; allo stesso modo, viene raccomandato un controllo della funzione epatica e dell'emocromo inizialmente ogni 3-4 settimane. La funzione tiroidea (TSH, FT4), l'assetto lipidico e, negli uomini, il testosterone assieme alla Sex Hormon Binding Globulin (SHBG) devono essere dosati ogni 3-4 mesi, la renina ogni 6 mesi (Tabella 1).

Durante il trattamento, frequente è la comparsa di disturbi gastrointestinali (nausea, vomito, diarrea), soprattutto inizialmente quando viene progressivamente incrementata la dose giornaliera; l'aumento delle gamma-GT è invece atteso e non riveste significato di rilievo, mentre il farmaco deve essere sospeso quando il livello delle transaminasi supera 5 volte quello basale. Molto comuni sono le alterazioni della funzione tiroidea (ipotiroidismo centrale), la comparsa di ipercolesterolemia, di ipogonadismo primitivo e ginecomastia negli uomini. Abbastanza frequente è anche il riscontro di leucopenia e, in misura minore, di anemia e trombocitopenia. Disturbi indicativi di coinvolgimento del SNC, quali confusione mentale, sonnolenza, letargia, sono comuni quando i livelli plasmatici di mitotane superano i $20 \mathrm{mg} / \mathrm{L}$.

Le terapie sostitutive di pertinenza endocrina devono essere intraprese quando vi si presenti la necessità per migliorare la qualità di vita dei pazienti e aumentare la compliance al trattamento.

In particolare, la terapia sostitutiva con L-tiroxina va iniziata non appena vi sia riscontro di ipotiroidismo; per il trattamento dell'ipercolesterolemia è da preferire l'utilizzo di statine non metabolizzate dal CYP450 3A4 (pravastatina, rosuvastatina); il testosterone può essere considerato nei pazienti con ipogonadismo sintomatico, tenendo però presente che l'azione inibente esercitata dal mitotane sulla 5-alfa- reduttasi potrebbe ridurne l'efficacia [2]. Nei pazienti che sviluppano ginecomastia è indicato il trattamento con diidrotestosterone (DHT), disponibile in formulazione gel al $2,5 \%$ per uso topico, in quanto non suscettibile dell'azione della 5-alfa-reduttasi [6]. Infine, in caso di compromissione della funzione midollare è necessario sospendere il farmaco ed effettuare una valutazione ematologica al fine di stabilire la necessità di somministrare fattori mielostimolanti.

Poiché il mitotane si accumula nel tessuto adiposo, i pazienti dovranno sottoporsi periodicamente al dosaggio della mitotanemia anche una volta sospesa la terapia, fino a quando i livelli plasmatici del farmaco non saranno più dosabili. Allo stesso modo, anche gli indici ormonali e metabolici sopracitati dovranno essere monitorati per poter sospendere al momento opportuno le terapie intraprese per il controllo degli effetti collaterali. Il trattamento steroideo sostitutivo verrà interrotto solo una volta ripristinata la funzionalità dell'asse ipotalamo-ipofisi-surrene.

\section{Conclusioni}

Ad oggi, la terapia adiuvante con mitotane rappresenta una pietra miliare nel trattamento dei pazienti con ACC. Il follow-up durante il periodo di assunzione del farmaco e alla sua sospensione deve essere affidato a endocrinologi e oncologi esperti della patologia al fine di garantire un'adeguata gestione dei pazienti e degli effetti avversi.

Funding Note Open access funding provided by Università degli Studi di Firenze within the CRUI-CARE Agreement.

Nota della casa editrice Springer Nature rimane neutrale in riguardo alle rivendicazioni giurisdizionali nelle mappe pubblicate e nelle affiliazioni istituzionali.

Open Access This article is licensed under a Creative Commons Attribution 4.0 International License, which permits use, sharing, adaptation, distribution and reproduction in any medium or format, as long as you give appropriate credit to the original author(s) and the source, provide a link to the Creative Commons licence, and indicate if changes were made. The images or other third party material in this article 
are included in the article's Creative Commons licence, unless indicated otherwise in a credit line to the material. If material is not included in the article's Creative Commons licence and your intended use is not permitted by statutory regulation or exceeds the permitted use, you will need to obtain permission directly from the copyright holder. To view a copy of this licence, visit http://creativecommons.org/licenses/by/4.0/.

\section{Bibliografia}

1. Kerkhofs TM, Verhoeven RH, Van der Zwan JM et al (2003) Adrenocortical carcinoma: a population-based study on incidence and survival in the Netherlands since 1993. Eur J Cancer 49(11):2579-2586

2. Fassnacht M, Dekkers OM, Else T et al (2018) European Society of Endocrinology Clinical Practice Guidelines on the management of adrenocortical carcinoma in adults, in collaboration with the Euro- pean Network for the Study of Adrenal Tumors. Eur J Endocrinol 179(4):G1-G46

3. Terzolo M, Angeli A, Fassnacht M et al (2007) Adjuvant mitotane treatment for adrenocortical carcinoma. N Engl J Med 356(23):2372-2380

4. Bergenstal DM, Hertz R, Lipsett MB, Moy RH (1960) Chemotherapy of adrenocortical cancer with o,p'DDD. Ann Intern Med 53:672-682

5. Veytsman I, Nieman L, Fojo T (2009) Management of endocrine manifestations and the use of mitotane ad a chemotherapeutic agent for adrenocortical carcinoma. J Clin Oncol 27(27):4619-4629

6. Chortis V, Taylor AE, Schneider P et al (2013) Mitotane therapy in adrenocortical cancer induces CYP3A4 and inhibits 5alfareductase, explaining the need for personalized glucocorticoid and androgen replacement. J Clin Endocrinol Metab 98(1):161-171

7. Terzolo M, Daffara F, Ardito A et al (2014) Management of adrenal cancer: a 2013 update. J Endocrinol Invest 37(3):207-217 